\title{
Knowledge Enrichment Analysis for Human Tissue- Specific Genes Uncover New Biological Insights
}

\author{
Xiu-Jun Gong ${ }^{1 *}$, Hua Yu${ }^{1}$, Chun-Bai Yang ${ }^{1}$, Yuan-Fang $\mathrm{Li}^{2}$ \\ ${ }^{1}$ School of Computer Science and Technology, Tianjin University, Weijin Rd No. 92, Nankan, \\ Tianjin, 300072, China \\ ${ }^{2}$ Clayton School of IT, Faculty of Information Technology,Monash University, Wellington Rd, \\ Clayton, Vic 3800, Australia
}

\begin{abstract}
Summary
The expression and regulation of genes in different tissues are fundamental questions to be answered in biology. Knowledge enrichment analysis for tissue specific (TS) and housekeeping (HK) genes may help identify their roles in biological process or diseases and gain new biological insights.In this paper, we performed the knowledge enrichment analysis for 17,343 genes in 84 human tissues using Gene Set Enrichment Analysis (GSEA) and Hypergeometric Analysis (HA) against three biological ontologies: Gene Ontology (GO), KEGG pathways and Disease Ontology (DO) respectively.The analyses results demonstrated that the functions of most gene groups are consistent with their tissue origins. Meanwhile three interesting new associations for HK genes and the skeletal muscle tissuegenes are found. Firstly, Hypergeometric analysis against KEGG database for HK genes disclosed that three disease terms (Parkinson's disease, Huntington's disease, Alzheimer's disease) are intensively enriched.Secondly, Hypergeometric analysis against the KEGG database for Skeletal Muscle tissue genes shows that two cardiac diseases of "Hypertrophic cardiomyopathy (HCM)" and "Arrhythmogenic right ventricular cardiomyopathy (ARVC)" are heavily enriched, which are also considered as no relationship with skeletal functions.Thirdly, "Prostate cancer" is intensively enriched in Hypergeometric analysis against the disease ontology (DO) for the Skeletal Muscle tissue genes, which is a much unexpected phenomenon.
\end{abstract}

\section{Introduction}

Exploring the tissue-specificity of gene expressions and their regulations is always a main theme in biology [1].Housekeeping (HK) genes are constitutively expressed in all tissues while Tissue Specific (TS) genes are expressed at a much higher level in a single tissue type than in others. HKgenes serve as valuable experimental controls in gene and protein expression experiments, while TS genes tend to represent distinct physiological processes and are frequent candidates for biomarkers or drug targets [1 - 3].

In past years, many investigations have been conducted the expression and regulation mechanisms of $\mathrm{HK}$ and TS genes [4 - 8]. Emerging technologies including Microarray technology [9], Expressed Sequence Tag(EST) [10], Serial Analysis of Gene Expression (SAGE) [10], and Chip-Seq technology [11] enable the analysis of expression patterns of HK and TS genes at genome scale. The analysis for genomic and epigenetic features of two groups of genes may shed light on the mechanisms by which cells maintain basic and tissuespecific functions [10], [12 - 17]. Also several tissue specific databases such as TiGER[18], TiProD [19], TissueInfo [20], TissueDistributionDBs [21] and TiSGeD [22] provide web

\footnotetext{
* To whom correspondence should be addressed. Email: gongxj@tju.edu.cn
} 
services to extract the TS and HK genes and their genomic features by predefined threshold values. However, there is no widely accepted criterion to judge which gene is expressed in a specific tissue only by the expression patterns. More, the integrative behaviors of extracted groups of genes, for instance, the biological functions and disease associations with these extracted groups of genes, have not been extensively studied. Such information is useful in the identification of HK and TS genes but also in the discovery of new biological insights.

The gene set enrichment analysis aims to seeking enrichment with a group of genes to assess whether subsets of this group show statistical significance of biological attributes against backgroundknowledge.Hypergeometric analysis and GSEAare two commonly used algorithms to acquire such enrichment terms and assess statistical significance. Hypergeometric analysis [23] calculates the possibility of the enrichment of one biological term in a target set against the background set with the assumption that genes are sampled from a hypergeometric distribution. GSEA [24] relies on phenotype labels to distinguish gene expression patterns under different conditions with the consideration of degree of change in expression values. Both of them have been widely used for the gene set enrichment analysis of gene groups. Dezso et al. [25] conducted enrichment analysis of HK and TS gene sets using the hypergeometric distributionand Gene Set Enrichment Analysis (GSEA) algorithms to determine enrichment across four functional ontologies: canonical pathway maps, gene ontology (GO) processes, GeneGo (GG) process networks, and diseases.Their analysis showed that thebiological functions of HK and TS are consistent with tissue origin.

In this paper we performed the knowledge enrichment analysis for 17,343 genes in 84 human tissues using Gene Set Enrichment Analysis (GSEA) and Hypergeometric Analysis (HA) against three biological ontologies: Gene Ontology (GO), KEGG pathways and Disease Ontology (DO).The analyses demonstrated that the functions of most gene groups are consistent with their tissue origins. Meanwhile three interesting associations for HK genes and the skeletal muscle tissue genes from the analysis might uncover new biological insights.

\section{Methods}

\subsection{Data sources and pre-processing}

We used the Human U133A/GNF1H Gene expression data to determine housekeeping and tissue-specific genes. The whole gene expression data and GNF1H annotation files linking Ensembl transcripts to their representative probes were downloaded from GNF (Genomics Institute of the Novartis Research Foundation) and U133A chip annotation files were downloaded from the Affymetrix website. The dataset contains 33,698 genes across 84 human tissues. To perform the enrichment analysis, all the genes in the dataset are unified as gene symbols. The probe sets which have no corresponding gene symbols are removed, and expression values with redundant probe IDs are averaged. Finally, 17,343 genes across 84 tissues are remained for further analysis.

Based on the purified gene expression dataset, we identified HK and TS genes. A gene whose expression value is no less than 3 standard deviations above the mean expression value in the give tissueis regarded as the TS gene. A gene whose expression value is more than 60 in no less than 75 tissues is regarded as the HK gene. Finally, we obtained1382 HK genes and 5,721 tissue-specific geneswith average 68.1 genes per tissue.

\subsubsection{Knowledgebase preparations}

To perform the enrichment analysis, we used three ontologies: Gene Ontology (GO) BP processes, KEGG Pathway, and Disease Ontology (DO). 
The GO unifies the representation of gene and gene product attributes across all species using controlled vocabularies. It covers there domains: Cellular Component (CP), Molecular Function (MF) and Biological Process (BP). BP processconsists of operations or sets of molecular events with a defined beginning and end, pertinent to the functioning of integrated living units: cells, tissues, organs, andorganisms. This component is used to perform the biological process enrichment analysis in our study.

KEGG is a collection of five online databases dealing with genomes, enzymatic pathways, and biological chemicals. It describes networks of molecular interactions in the cells, and variants of them specific to particular organisms. We use the KEGG PATHAY database for the biological pathway enrichment analysis.

Disease ontology (DO)organises disease conceptsinto a directed acyclic graph (DAG) just like GO. Its mission is to integrate biomedical data for human diseases. DO has been used to annotate the human genome and show better performance than other current human disease annotations. We downloaded the DO from http://www.obofoundry.org/cgibin/detail.cgi?id=disease_ontology and compiled a map between genes and terms in DO. The Table 1 lists versions and sources of the three ontologies.

Table 1: knowledgebase and their versions

\begin{tabular}{|l|l|}
\hline Knowledgebase & version \\
\hline GO BP & c5.bp.v3.0.symbols.gmt \\
\hline KEGG Pathway & c2.cp.kegg.v3.0.symbols.gmt \\
\hline Disease Ontology (DO) & Access in May, 2011 \\
\hline
\end{tabular}

\subsubsection{Enrichment analysis}

The enrichment analysis seeks to infer if a group of genes show statistical significance of biological attributes against the background knowledge.

We used the p-value as the enrichment levels of the HK genes and tissue-specific genes in Hypergeometric Analysis. P-value is calculated according to formula (1).

$$
p(k)=\sum_{i=k}^{D} p(i, M, n, N)
$$

Where $\mathrm{N}$ and $\mathrm{n}$ are the numbers of genes in the background database and targeted gene set respectively, $\mathrm{M}$ and $\mathrm{i}$ are the numbers of genes labelled by a give biological term in the background database and targeted gene set respectively. The value $p(i, M, n, N)$ follows the Hypergeometric distribution:

$p(i, M, n, N)=\frac{\left(\begin{array}{c}M \\ i\end{array}\right)\left(\begin{array}{c}N-M \\ n-i\end{array}\right)}{\left(\begin{array}{l}N \\ n\end{array}\right)}$

The p-value gives probability of having at least $\mathrm{k}$ marked elements in a sample of size $\mathrm{n}$ by random selection. 
In the Hypergeometric Analysis, we used GeneCodis2 (http://genecodis.dacya.ucm.es/) for KEGG Pathway and GO BP, and fundo (http://django.nubic.northwestern.edu/fundo/) for the Disease Ontology (DO).

We used GSEA from http://www.broadinstitute.org/gsea/index.jsp for Gene Set Enrichment Analysis. The GO BP and KEGG pathway gene sets are both obtained from MSigDB. We compiled an annotation file for the Disease Ontology (DO) enrichment analysis.

\section{$3 \quad$ Results}

\subsection{List of housekeeping genes and tissue-specific genes}

We set up a fixed cutoff value to determine whether a gene is expressed in a specific tissue for the gene expression dataset; see the method part for details. Finally, 1382 housekeeping genes and 5,721 tissue-specific geneswith average 68.1 per tissue are identified. See Table 2 for more details.

\subsection{Knowledge enrichment analysis for Housekeeping genes}

We performed the HA against GO BP, KEGG Pathway and DO, for the enriched terms and their distributions (see Table 3 and Figure 1 respectively).

The results produced by $\mathrm{HA}$ for all the 84 tissues are accessible from http://cs.tju.edu.cn/faculty/gongxj/specifictome/HA.htm.

For the GO biological process, the top 10 terms can be classified approximatelyin three groups. The first group is related to central dogma with terms like "translational elongation", "translation", "RNA splicing" and "mRNA processing". These biological processes are vital in transmission of gene information and fulfilment of cells' functions. The second group is related to metabolism with terms like "electron transport chain", "mitochondrial ATP synthesis coupled proton transport", "mitochondrial electron transport, NADH to ubiquinone". Dezso et al. have performed a similar analysis of human HK and TS genes in 31 tissues [25]. In their analysis, metabolic terms concerned with electron transport, ATP synthesis are the most enriched ones. And for the third group, terms like "regulation of ubiquitin-protein ligase activity during mitotic cell cycle" and "anaphase-promoting complex-dependent proteasomal ubiquitin-dependent protein catabolic process" are involved. They are related to mitosis which is also fundamental to every tissue. All three groups are relevant to fundamental functions that maintain cells.

For the KEGG Pathway procedure, the enriched terms "Ribosome", "Oxidative phosphorylation", "Spliceosome" can be explained by its fundamental functions in metabolism or mitosis of HK genes. However a very interesting association is that three diseases terms: Parkinson's disease", "Huntington's disease" and "Alzheimer's disease" are intensively enriched. These three are typical neurodegenerative diseases caused by malformed proteins. Seemingly they are related with brain and nervous system and have nothing to do with the functions of housekeeping genes. However, [26] et al'showed that "A growing body of evidence now suggests that dysfunctionof autophagy causes accumulation of abnormal proteins and/or damaged organelles. Such accumulation has been linked to synaptic dysfunction, cellular stress and neuronal death.” Also they mentioned these three diseases are related to chronic nervous system disorders caused by dysfunction of autophagy. Therefore, we hypothesizethat the autophagy function of housekeeping genes might result in the three diseases. 
Table 2: The numbers of housekeeping genes and tissue-specific genes

\begin{tabular}{|c|c|c|c|c|c|}
\hline Tissue name & \# genes & Tissue name & \# genes & Tissue name & \# genes \\
\hline Housekeeping & 1382 & 721_B_lymphoblasts & 475 & Adipocyte & 57 \\
\hline AdrenalCortex & 32 & Adrenalgland & 20 & Amygdala & 57 \\
\hline Appendix & 38 & AtrioventricularNode & 56 & $\begin{array}{l}\text { BDCA4+_DentriticCel } \\
\text { ls }\end{array}$ & 106 \\
\hline Bonemarrow & 23 & $\begin{array}{l}\text { BronchialEpithelialCel } \\
\text { ls }\end{array}$ & 88 & CD105+_Endothelial & 46 \\
\hline CD14+_Monocytes & 27 & $\begin{array}{l}\text { CD19+_BCells(neg._s } \\
\text { el.) }\end{array}$ & 95 & CD33+_Myeloid & 114 \\
\hline CD34+ & 133 & CD4+_Tcells & 29 & CD56+_NKCells & 186 \\
\hline $\begin{array}{l}\text { CD71+_EarlyErythroi } \\
\text { d }\end{array}$ & 226 & CD8+_Tcells & 60 & CardiacMyocytes & 105 \\
\hline Caudatenucleus & 27 & Cerebellum & 19 & CerebellumPeduncles & 47 \\
\hline CiliaryGanglion & 73 & CingulateCortex & 17 & $\begin{array}{l}\text { Colorectaladenocarcin } \\
\text { oma }\end{array}$ & 82 \\
\hline DorsalRootGanglion & 22 & FetalThyroid & 10 & Fetalbrain & 93 \\
\hline Fetalliver & 36 & Fetallung & 11 & GlobusPallidus & 19 \\
\hline Heart & 178 & Hypothalamus & 20 & Kidney & 63 \\
\hline $\begin{array}{l}\text { Leukemia_chronicMye } \\
\text { logenousK-562 }\end{array}$ & 32 & $\begin{array}{l}\text { Leukemia_promyelocy } \\
\text { tic-HL-60 }\end{array}$ & 4 & $\begin{array}{l}\text { Leukemialymphoblasti } \\
\text { c(MOLT-4) }\end{array}$ & 16 \\
\hline Liver & 313 & Lung & 83 & Lymphnode & 8 \\
\hline $\begin{array}{l}\text { Lymphoma_burkitts(D } \\
\text { audi) }\end{array}$ & 45 & $\begin{array}{l}\text { Lymphoma_burkitts(R } \\
\text { aji) }\end{array}$ & 117 & MedullaOblongata & 6 \\
\hline OccipitalLobe & 8 & OlfactoryBulb & 33 & Ovary & 13 \\
\hline Pancreas & 30 & PancreaticIslet & 43 & ParietalLobe & 14 \\
\hline Pituitary & 50 & Placenta & 173 & Pons & 22 \\
\hline PrefrontalCortex & 141 & Prostate & 77 & Salivarygland & 24 \\
\hline SkeletalMuscle & 212 & Skin & 35 & SmoothMuscle & 80 \\
\hline Spinalcord & 24 & SubthalamicNucleus & 30 & $\begin{array}{l}\text { SuperiorCervicalGangl } \\
\text { ion }\end{array}$ & 233 \\
\hline TemporalLobe & 5 & Testis & 82 & TestisGermCell & 43 \\
\hline TestisIntersitial & 90 & TestisLeydigCell & 24 & $\begin{array}{l}\text { TestisSeminiferousTub } \\
\text { ule }\end{array}$ & 13 \\
\hline Thalamus & 35 & Thymus & 20 & Thyroid & 112 \\
\hline Tongue & 51 & Tonsil & 7 & Trachea & 28 \\
\hline TrigeminalGanglion & 51 & Uterus & 56 & UterusCorpus & 35 \\
\hline WholeBlood & 138 & Wholebrain & 56 & colon & 50 \\
\hline pineal_day & 49 & pineal_night & 62 & retina & 92 \\
\hline small_intestine & 66 & & & & \\
\hline
\end{tabular}




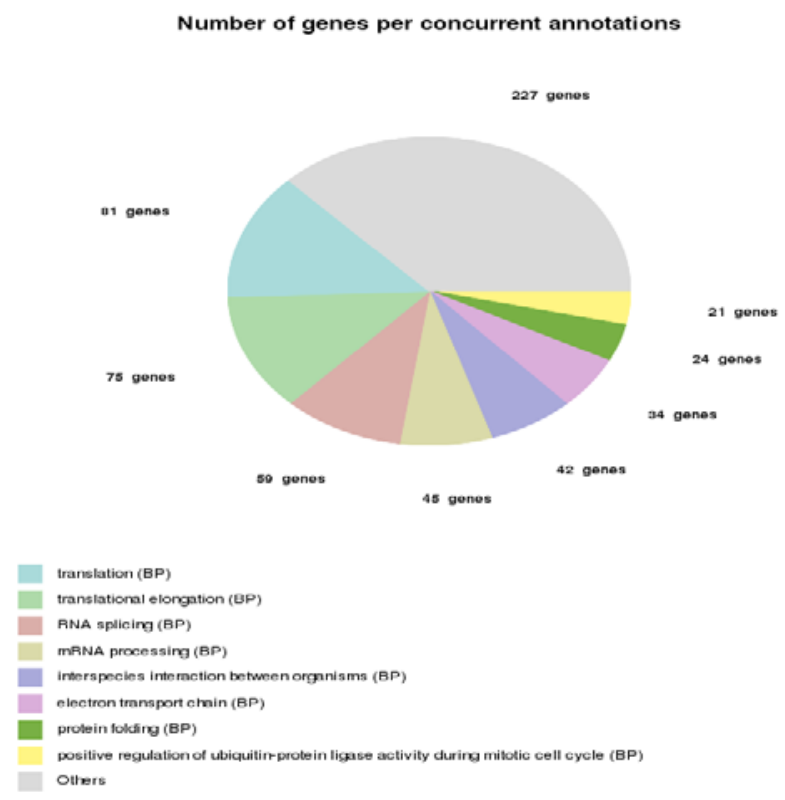

Figure 1: Number of genes per concurrent annotation in hypergeometric for GO biological process (generated by GeneCodis2)

Table 3: Enrichment terms of housekeeping genes using hypergeometric analysis

\begin{tabular}{|c|c|c|}
\hline GO biological process & KEGG pathway & Disease ontology \\
\hline $\begin{array}{l}\text { translational elongation } \\
\text { translation } \\
\text { electron transport chain } \\
\text { RNA splicing } \\
\text { mitochondrial ATP synthesis } \\
\text { coupled proton transport } \\
\text { mitochondrial ATP synthesis } \\
\text { coupled proton transport } \\
\text { positive regulation of ubiquitin- } \\
\text { protein ligase activity during } \\
\text { mitotic cell cycle } \\
\text { negative regulation of ubiquitin- } \\
\text { protein ligase activity during } \\
\text { mitotic cell cycle } \\
\text { mRNA processing }\end{array}$ & $\begin{array}{l}\text { Ribosome } \\
\text { Parkinson's disease } \\
\text { Huntington's disease } \\
\text { Oxidative phosphorylation } \\
\text { Alzheimer's disease } \\
\text { Spliceosome } \\
\text { Proteasome } \\
\text { Cardiac muscle contraction } \\
\text { Pathogenic Escherichia coli } \\
\text { infection } \\
\text { Antigen processing and } \\
\text { presentation }\end{array}$ & $\begin{array}{l}\text { Cancer } \\
\text { Colon Cancer } \\
\text { Prostate Cancer } \\
\text { Brain Tumor } \\
\text { Down syndrome } \\
\text { Pancreas cancer } \\
\text { Nasopharyngeal cancer } \\
\text { Breast cancer } \\
\text { Embryoma } \\
\text { Pancreas disease }\end{array}$ \\
\hline
\end{tabular}

For the Disease Ontology (DO) procedure, cancers and tumours take up most of highly enriched diseases. Cancer is caused by uncontrolled growth of cells which are related to the fundamental cellular functions like mitosis, autophagy and metabolism. See Figure 2 for more details.

\subsection{Knowledge enrichment analysis for TS genes}

We performedknowledge enrichment analysis for 84 human tissues using HA and GSEA against GO, KEGG pathway and DO. The functional annotations mostly are in consistence with their corresponding tissue's functions. 
Because of the page limitation, we list ten top-scored terms for skeletal muscle and retina tissues in Table 4.

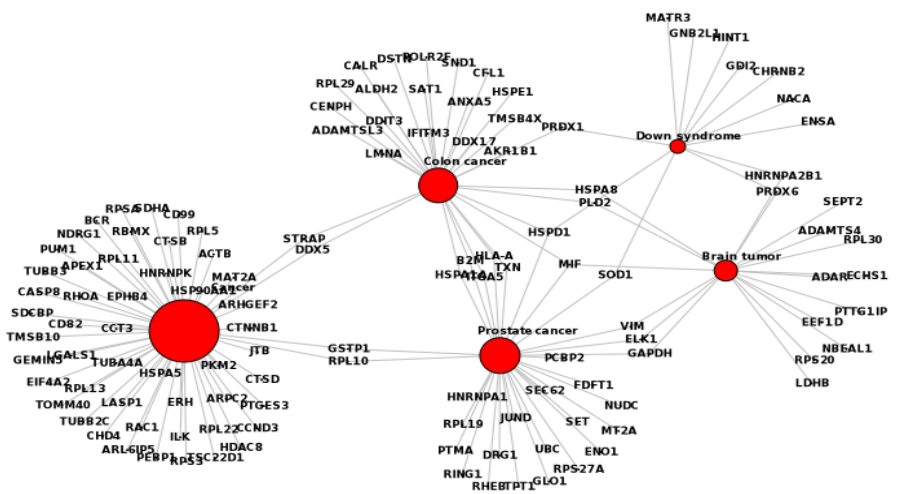

Figure 2: The mapping between genes and diseases in HA for DO enrichment analysis generated by FunDO.

Table 4: Enriched terms in HA and GSEA procedures for skeletal muscle and retina tissues

\begin{tabular}{|c|c|c|c|}
\hline & & Skeletal Muscle & Retina \\
\hline $\begin{array}{l}0 \\
0 \\
\sigma \\
0 \\
0\end{array}$ & $\stackrel{I}{D}$ & $\begin{array}{l}\text { muscle contraction } \\
\text { skeletal muscle contraction } \\
\text { muscle organ development } \\
\text { positive regulation of fast-twitch skeletal muscle fiber } \\
\text { contraction } \\
\text { regulation of striated muscle contraction } \\
\text { striated muscle contraction }\end{array}$ & $\begin{array}{l}\text { visual perception } \\
\text { response to stimulus } \\
\text { phototransduction, visible light } \\
\text { phototransduction } \\
\text { protein-chromophore linkage } \\
\text { melanin biosynthetic process } \\
\text { eye pigment biosynthetic process } \\
\text { melanin biosynthetic process from tyrosine } \\
\text { positive regulation of rhodopsin gene expression } \\
\text { regulation of rhodopsin gene expression }\end{array}$ \\
\hline
\end{tabular}




\begin{tabular}{|c|c|c|c|}
\hline & $\overbrace{\mathbb{D}}^{\mathrm{M}}$ & $\begin{array}{l}\text { neuroactive ligand receptor interaction } \\
\text { cytokine cytokine receptor interaction } \\
\text { tight junction } \\
\text { calcium signaling pathway } \\
\text { pentose phosphate pathway } \\
\text { heparansulfate biosynthesis } \\
\text { maturity onset diabetes of the young } \\
\text { carbon fixation } \\
\text { jak stat signaling pathway } \\
\text { alpha linolenic acid metabolism }\end{array}$ & $\begin{array}{l}\text { tyrosine metabolism } \\
\text { inositol phosphate metabolism } \\
\text { phenylalanine metabolism } \\
\text { beta alanine metabolism } \\
\text { TGF beta signaling pathway } \\
\text { retinol metabolism } \\
\text { sphingolipid metabolism } \\
\text { phosphatidylinositol signaling system } \\
\text { melanogenesis } \\
\text { histidine metabolism }\end{array}$ \\
\hline \multirow{2}{*}{ 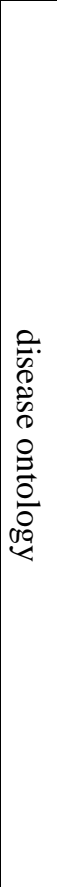 } & $\stackrel{T}{D}$ & $\begin{array}{l}\text { Prostate cancer } \\
\text { "Myopathies, Nemaline" } \\
\text { Rhabdomyosarcoma } \\
\text { Vaccinia } \\
\text { Muscular atrophy } \\
\text { Epilepsy } \\
\text { Drug abuse } \\
\text { Amyotrophic lateral sclerosis } \\
\text { Myopathy } \\
\text { Mucocutaneous lymph node syndrome }\end{array}$ & $\begin{array}{l}\text { Retinitis pigmentosa } \\
\text { Retinal disease } \\
\text { Uveomeningoencephalitic syndrome } \\
\text { Amaurosiscongenita of leber I } \\
\text { Macular degeneration } \\
\text { Eye disease } \\
\text { Glaucoma } \\
\text { Melanoma } \\
\text { Neoplasm metastasis } \\
\text { Uveitis }\end{array}$ \\
\hline & 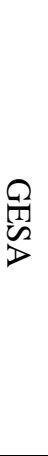 & $\begin{array}{l}\text { myopathy } \\
\text { premature ovarian failure } \\
\text { attention deficit hyperactivity disease } \\
\text { muscular dystrophy } \\
\text { myelofibrosis } \\
\text { disease of metabolism } \\
\text { heart failure } \\
\text { rhabdomyosarcoma } \\
\text { thyroid neoplasm } \\
\text { glucose intolerance }\end{array}$ & $\begin{array}{l}\text { retinitis pigmentosa } \\
\text { age related macular degeneration } \\
\text { retinal degeneration } \\
\text { glaucoma } \\
\text { vitiligo } \\
\text { retinal disease } \\
\text { autosomal dominant polycystic kidney } \\
\text { primary open anle glaucoma } \\
\text { hypercholesterolemia } \\
\text { basal cell carcinoma }\end{array}$ \\
\hline
\end{tabular}

For the GO BP procedure, the top scored terms in skeletal muscle are all behaviours critical to skeletal muscle. In HA, muscle contraction is dominated. However, the results of GSEA procedure are more diversified,ranging from muscle development, differentiation, and ion transport tosignalling. Ion channels are critical in regulation of muscle behaviours. The enrichment intensity for ion related behaviours is in accordance with this statement.

Hypergeometric analysis against the KEGG databases for the Skeletal Muscle tissue shows that "Hypertrophic cardiomyopathy (HCM)" and "Arrhythmogenic right ventricular cardiomyopathy (ARVC)" areheavily enriched, which are considered as having no relationship with skeletal functions. These may result in the shared functions between the skeletal and cardiac muscles.

For the DO procedure, the most enriched terms of for skeletal muscle tissue genes in both methods are quite reasonable disorders related to skeletal muscle. "Myopathy", "Rhabdomyosarcoma", "muscular atrophy" etc. are all typical skeletal muscle diseases. In HA, the term "prostate cancer" has the highest score which is quite unexpected. However, a common function between skeletal muscle and prostate is that they're both androgenregulated human tissues. Several studies focused on the effect of androgen on tissues such as skeletal muscle and prostate. Kumar R et al. pointed out in their study [27] that androgens are involved in the development of these tissues. And one regulator of androgens, namely the androgen receptor(AR) has association with prostate cancer. This is a potential reason for the enriched disease"prostate cancer" in skeletal muscle analysis.

The analysis results for the retina tissue are consistent with the one done by Dezaso et al. For GO BP, enriched terms can be classified in two groups. One includes the visual perception 
terms including phototransduction, detection and response to stimulus. The other is about retina pigment biosynthesis. The KEGG pathway result intensively concentrates on the biosynthesis of pigments and amino acids which are related to retina. Disease ontology analysis result agrees with the intuition which shows the connection with eye diseases like glaucoma and uveitis.

\section{Discussion}

\subsection{Identification of housekeeping and tissue-specific genes}

As for the identification of HK and TS genes based on expression datasets, there are several methods available. Dezso et al. used a 10 fold signal noise ratio criterion [25]. Hsiao et al. used present or absent calls to determine its presence [28]. Tu et al. and Su et al. used cutoff values to filter t HK and TS genes [9], [12]. We use a similar method with Su et al. and try several cutoff values to classify the two groups of genes. The distributions of the number of genes along with the number of tissues that the given genes are classified using cutoff values 40, 60, and 90 are drawn in Figure 3. Compared with the classification results of other researchers, the ideal distribution should be higher on both ends and lower in the middle region.
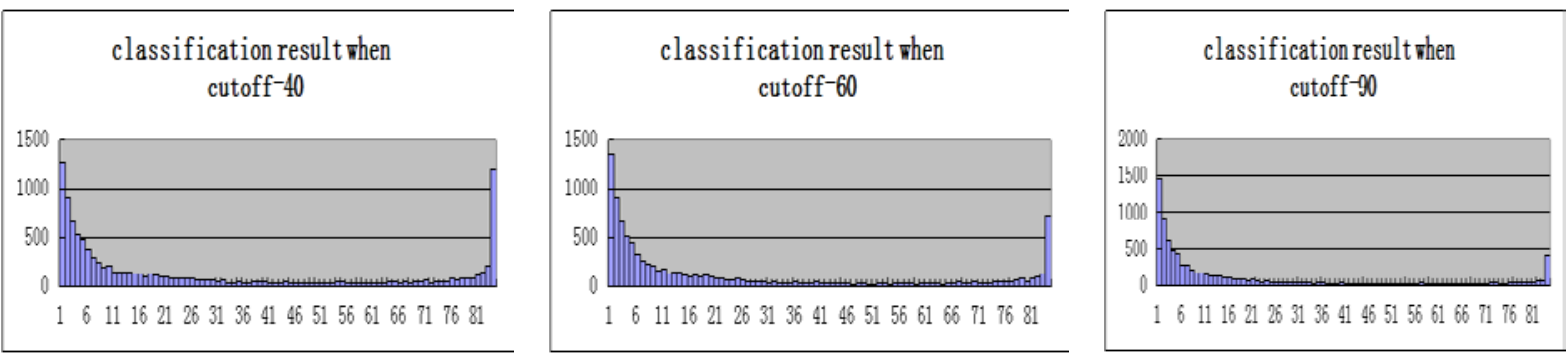

Figure 3: Relationship between the number of genes and the number of tissues that a given gene is classified using cutoff values 40,60 and 90 .

We took 60 as the cutoff value for HK gene classification. The genes with expression value more than 60 in no less than 75 tissues are considered as housekeeping gene. 1,382 out of the 17,343 genes are identified which correspond to the $10 \%$ estimation and also show their reasonableness in the functional analysis [12]. This criterion is not applicable to the identification of TS genes, because TS genes should be highly and uniquely expressed in a given tissue. There are genes that express on average much higher than others in all tissues. This results in the high variance of expression values of different genes in a tissue, while the expression values of a gene in different tissues tend to be less fluctuant. Wetake 3 standard deviations above the mean expression value of genes to be the cutoff for TS gene classifications.

\subsection{Complementary results from Hypergeometric analysis and GSEA}

Hypergeometric analysis and GSEA method both have their own advantages. Hypergeometric analysis is the classical way for enrichment analysis. It requires users' specification of target genes which are usually gained by classifications on expression value.It is straightforward and has the strength in integrating researchers' prior knowledge outside the expression data. However, the enrichment results are unreliable when no enough evidencesareavailable. GSEAonly requires specification of labels to distinguish genes in different conditions. It also takes into account all genes' expression values and focuses on a prior defined gene sets like cell cycle.This difference makes Hypergeometric analysis more subjective while GSEA more 
objective. The incorporation of both algorithms gives us more comprehensive functional annotations.

We can see this complementation in the analysis results. The 3 standard deviation above mean is a quite stringent cutoff. In Hypergeometric analysis, tissues having few genes, usually less than 40, have very few enriched terms or even none. However, GSEA results are stable and independent of the choice of cutoff. For the stringent cutoff, Hypergeometric analysis results tend to be clustered on some highly significant functions while GSEA tend tobe ranged more widely.

\subsection{Disease ontology}

Disease ontology was initially releasedin 2003 as part of a project of Northwestern University. It has been demonstrated its usefulness in several experiments and has also given very reasonable enrichment terms for many tissues. Osborne et al. produced an annotation of human genome with DO terms using GeneRif database [29]. Their validation data set suggested a much higher recall rate and precision rate against the widely used Online Mendelian Inheritance in Man(OMIM) annotations.However, relatively little attention has been given to DO. We could only find 16 articles from Pubmed using the key word "disease ontology” searching. There're also very few tools availableto make use of DO.

\section{Conclusions}

Knowledge enrichment analyses for HK and TS genes show that most of functions for these two groups of genes are consistent with their tissue origins. However some of them show unexpected functions, which may be vital clues for further exploring their functions or the mechanisms of some diseases. Our experiments also demonstrated that GSEA method is preferred in the verification of consistent knowledge, while HA in the discovery of ones for groups of genes. Therefore GSEA and HA can be used complementarily for the analysis of groups of genes.

\section{Acknowledgements}

This research is partly supported by the Natural Science Funding of China under grant number 61170177and innovation funding of Tianjin University.

\section{References}

[1] J. Zhu, F. He, S. Hu, and J. Yu. On the nature of human housekeeping genes. Trends Genet, 24 (10):481-4, 2008.

[2] N. Rosenfeld et al.. MicroRNAs accurately identify cancer tissue origin. Nat. Biotechnology, 26 (4):462-9, 2008.

[3] J. Gilbert, M. Haber, S. B. Bordow, G. M. Marshall, and M. D. Norris. Use of TumorSpecific Gene Expression for the Differential Diagnosis of Neuroblastoma from Other Pediatric Small Round-Cell Malignancies. Am. J. Pathol., 155 (1):17-21, 1999.

[4] X. Yu, J. Lin, D. J. Zack, and J. Qian. Computational analysis of tissue-specific combinatorial gene regulation: predicting interaction between transcr iption factors in human tissues. Nucleic Acids Research, 34 (17):4925-36, 2006.

[5] K. E. Kouadjo, Y. Nishida, J. F. Cadrin-girard, M. Yoshioka, and J. St-amand. Housekeeping and tissue-specific genes in mouse tissues. BMC Genomics, 8:127, 2007. 
[6] C. Zhang, Z. Zhang, J. Castle, S. Sun, and J. Johnson. Defining the regulatory network of the tissue-specific splicing factors Fox-1 and Fox-2. Genes Dev., 22 (18):2550-63, 2008.

[7] D. Kuzmin et al.. Novel strong tissue specific promoter for gene expression in human germ cells. BMC Biotechnology, 10:58, 2010.

[8] T. Shlomi, M. N. Cabili, M. J. Herrgård, B. Ø. Palsson, and E. Ruppin. Network-based prediction of human tissue-specific metabolism. Nature Biotechnology, 26 (9):1003-10, 2008.

[9] A. I. Su et al.. Large-scale analysis of the human and mouse transcriptomes. Proc. Natl. Acad. Sci. U.S.A., 99 (7):4465-70, 2002.

[10] V. E. Velculescu, L. Zhang, B. Vogelstein, and K. W. Kinzler. Serial analysis of gene expression. Science, 270 (5235):484-7, 1995.

[11] A. Visel et al. ChIP-seq accurately predicts tissue-specific activity of enhancers. Nature, 457 (7231):854-8, 2009.

[12] Z. Tu, L. Wang, M. Xu, X. Zhou, T. Chen, and F. Sun. Further understanding human disease genes by comparing with housekeeping genes and other genes. BMC Genomics, 7:31, 2006.

[13] E. Schilling. Analysis of tissue-specific \& allele-specific DNA methylation. PhD thesis, University Hospital Regensburg, 2010.

[14] A. Grosso, A. Gomes, and N. Barbosa. Tissue-specific splicing factor gene expression signatures. Nucleic Acids Reserach, 36 (15):4823-32, 2008.

[15] E. T. Wang et al.. Alternative isoform regulation in human tissue transcriptomes, Nature, 456 (7221):470-6, 2008.

[16] J. Schug, W. Schuller, and C. Kappen. Promoter features related to tissue specificity as measured by Shannon entropy. Genome Biol., 6 (4):R33, 2005.

[17] F. Song et al. Tissue specific differentially methylated regions (TDMR): Changes in DNA methylation during development. Genomics, 93 (2):130-9, 2009.

[18] X. Liu, X. Yu, D. J. Zack, H. Zhu, and J. Qian. TiGER : A database for tissue-specific gene expression and regulation. BMC Bioinformatics, 9:271, 2008.

[19] X. Chen, J.-min Wu, K. Hornischer, A. Kel, and E. Wingender. TiProD: the tissuespecific promoter database. Nucleic Acids Research, 34 (Database issue):D104-7, 2006.

[20] L. Skrabanek and F. Campagne. TissueInfo: high-throughput identification of tissue expression profiles and specificity. Nucleic Acids Research, 29 (21):E102-2, 2001.

[21] S. Kogenaru, C. D. Val, A. Hotz-Wagenblatt, and K.H. Glatting. TissueDistributionDBs: a repository of organism-specific tissue-distribution profiles. Theoretical Chemistry Accounts, 125:651-658, 2010.

[22] S.-J. Xiao, C. Zhang, and Z.-L. Ji. TiSGeD: a database for tissue-specific genes. Bioinformatics, 26 (9):1273-5, 2010.

[23] L. A. Pennacchio, G. G. Loots, M. A. Nobrega, and I. Ovcharenko. Predicting tissuespecific enhancers in the human genome. Genome Research, 17 (2):201-11, 2007.

[24] A. Subramanian, P. Tamayo, V. K. Mootha, S. Mukherjee, and B. L. Ebert. Gene set enrichment analysis : A knowledge-based approach for interpreting genome-wide. Proc. Natl. Acad. Sci. U.S.A., 102 (43):15545-50, 2005.

[25] Z. Dezso et al.. A comprehensive functional analysis of tissue specificity of human gene expression. BMC Biology, 6:49, 2008.

[26] M. Xilouri and L. Stefanis. Autophagy in the central nervous system: implications for neurodegenerative disorders. CNS Neurol Disord Drug Targets, 9 (6):701-19, 2010. 
[27] R. Kumar, H. Atamna, M. N. Zakharov, S. Bhasin, S. H. Khan, and R. Jasuja. Role of the androgen receptor CAG repeat polymorphism in prostate cancer, and spinal and bulbar muscular atrophy. Life Sci., 88 (13-14):565-71, 2011.

[28] L. L. Hsiao et al.. A compendium of gene expression in normal human tissues. Physiol. Genomics, 7 (2):97-104, 2001.

[29] J. D. Osborne et al.. Annotating the human genome with Disease Ontology. BMC Genomics, 10 Suppl (1):S6, 2009. 